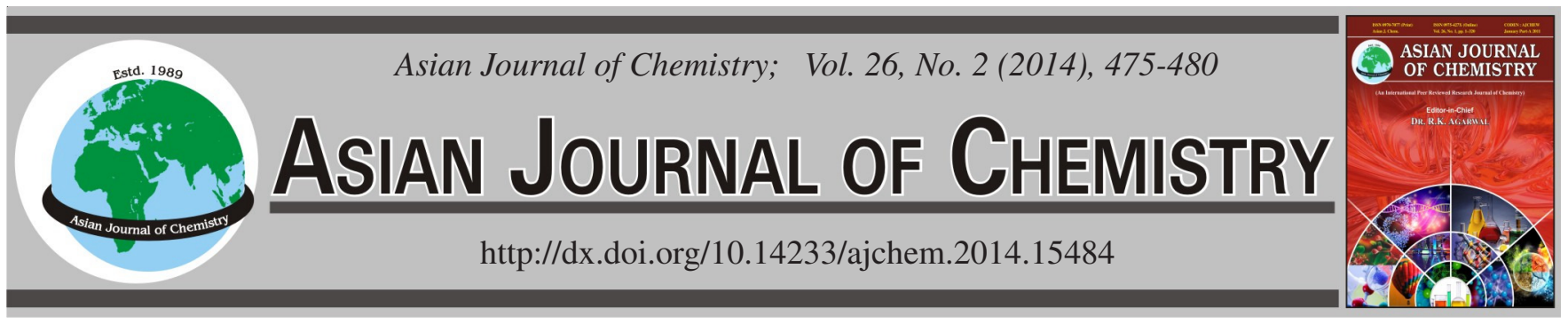

\title{
Study on Gas-Phase Mechanism of Chloroacetic Acid Synthesis by Catalysis and Chlorination of Acetic Acid
}

\author{
Jian-Wei Xue ${ }^{*}$, Jian-Peng Zhang, Bo Wu, Fu-Xiang Li and Zhi-Ping Lv
}

Research Institute of Special Chemicals, Taiyuan University of Technology, Taiyuan 030024, Shanxi Province, P.R. China

*Corresponding author: Fax: +86 351 6111178; Tel: +86 351 60105503; E-mail: xuejianwei@yeah.net

The process of acetic acid catalysis and chlorination for synthesizing chloroacetic acid can exist in not only gas phase but also liquid phase. In this paper, the gas-phase reaction mechanism of the synthesis of chloroacetic acid was studied. Due to the high concentration of acetic acid and the better reaction mass transfer in the liquid-phase reaction, the generation amount of the dichloroacetic acid was higher than that in the gas-phase reaction. Under the solution distillation, the concentration of acetyl chloride, whose boiling point is very low, was very high in the gas phase, sometimes even up to $99 \%$, which would cause the acetyl chloride to escape rapidly with the hydrogen chloride exhaust, so that the reaction slowed down. Therefore, series reactions occured easily in the gas-phase reaction causing the amount of the dichloroacetic acid to increase.

Keywords: Gas phase, Catalysis, Chlorination, Chloroacetic acid, Acetic acid.

\section{INTRODUCTION}

Chloroacetic acid is not only a fine chemical product but also an important intermediate in organic synthesis. The chloroacetic acid, which is widely used in pharmaceuticals, pesticides, dyes, paints and household chemicals industry, has played an important role in the national economy. Dichloroacetic acid will be generated inevitably during the synthesis of chloroacetic acid, which has brought great troubles to industrial production of chloroacetic acid. For example, it affects not only the yield but also the quality of chloroacetic acid products. Researchers had no systematic studying in gas phase part of chloroacetic acid synthesis by catalysis and chlorination of acetic acid. Therefore, dichloroacetic acid generation mechanism has been a hot research topic. And a method of controlling chloride side reaction (dichlorophenylamino side reaction) should be found from its reaction mechanism.

In 1923, Watson et al. found that the forming of olefinic alcohol existed in the acetic acid chlorination process. Then Sioli et al. ${ }^{1}$ proved that the course of acetic acid chlorination was an ion reaction and the enolization of acetyl chloride was the controlling step in the acetic acid chlorination process ${ }^{1}$.

Giuffre et $a l^{2}{ }^{2}$ suggested the following conclusions according to the experiment: the chlorination process of acetic acid included an enolization step, which was a control step of the acetic acid chloride reaction. It should be noted that the enolization step could be catalyzed by catalysts.
Martikainen et $a l .^{3}$ summed up the reaction mechanism that was consistent with a mechanism found by Sioli according to the system condition experiment and systematic theoretical analysis. Then deepen this mechanism to the acid-catalyzed and enolization of the acetyl chloride. Enolization of acetyl chloride was the controlling step of the reaction and chloroacetic acid could be self-catalyzed. Until now, an integral reaction mechanism has been drawn that the controlling step of this reaction mechanism was caused by the acid-catalyzed and enolization of the acetyl chloride, which was accepted by the majority ${ }^{3}$. So far, there has been no new breakthrough in the reaction mechanism of the acetic acid catalysis and chlorination.

However, many researchers have studied the mechanism of catalysis and chlorination of acetic acid. There were also a number of workers studying the kinetic conditions of experiments. For example, the data with first-order kinetics of acetic acid chlorination was described by Kumar and Gehlawat ${ }^{4}$ and the halogenation of carboxylic acids in the presence of chloro-sulphonic acid was studied by Ogata and co-work$\mathrm{ers}^{5,6}$. Therefore, various kinetic models of acetic acid that was catalyzed and chlorinated with different catalysts were established.

Salmi et al. ${ }^{7}$ reported the kinetic conditions of experiment of catalysis and chlorination of acetic acid and established the kinetic mathematical model using acetyl chloride as catalyst, concentrated sulfuric acid as co-catalyst. Maki-Arvela et al. ${ }^{8}$ 
also did the the contrast kinetic condition experiment of catalysis and chlorination of acetic acid and established the kinetic mathematical model respectively using chlorosulfonic acid and thionyl chloride as catalyst and summed up the kinetic model ${ }^{7,8}$. Salmi et al. ${ }^{9,10}$ reported the reaction mechanism in detail.

Cui et al. ${ }^{11}$ used the acetic acid for photochemical chlorination to prepare chloroacetic and studied its kinetics. Then the parallel-series kinetic model was put forward. Series reactions(depth chlorination of chloroacetic acid) and acetic acid parallel chlorination both simultaneously caused the dichloroacetic acid to generate.

Maki-Arvela et al. ${ }^{12}$ studied the mechanism of chlorination of acetic acid using acetyl chloride, chlorosulfonic acid, thionyl chloride as catalysts. According to the result, the generation amount of dichloroacetic acid increased with the increasing of the generation amount of chloroacetic acid under the condition of low conversion rate or moderate conversion rate; but the generation amount ratio of them was altered when using different catalysts. The generation rate of dichloroacetic acid increased under the condition of high conversion rate. As a consequence, this reaction was considered as a parallel reaction. However, some researchers believed that the intermediates of generating dichloroacetic acid should be dichloroacetyl chloride and the chlorination of acetyl chloride led to generating dichloroacetyl chloride. Therefore, this reaction should be series reaction.

In this study, the generation mechanism of dichloroacetic acid is widely concerned. The authors attempt to study gasphase reaction mechanism of the synthesis of chloroacetic acid and find the evidence that the generation rate of the dichloroacetic acid becomes faster at the late term of the chlorination reaction in order to guide specific production of the chloroacetic acid industry.

\section{EXPERIMENTAL}

The $99.9 \%$ pure chlorine gas is industrial grade; acetic acid (Merck 1455, Stock 33252, > $99 \%$ ) is the basic raw material for the synthesis of chloroacetic acid (Merck 142112, Stock A11482, $99 \%$ ); Both acetic anhydride (Merck 1456, Stock 36292, > $97 \%$ ) and acetyl chloride (Merck 1485, Stock $43262,>99 \%$ ) are catalysts for the chlorination reaction. In order to remove hydrogen chloride exhaust, sodium chloride and sodium hydroxide are of analytical grade; glycol solution as low the temperature medium of circulating pump is of analytical grade.

Fig. 1 is the gas-liquid reaction equipment. Gas liquid reaction equipment: (1) Chlorine rotameter; (2) Balanced feeder; (3) Drip valve; (4) Fixed bed chlorination reactor (glass, homemade, gas distribution with a sand core); (5) Glass spherical condenser; (6) Receiving flask of reaction solution; (7) Hot-water circulating pump; 8)Low-temperature circulating pump; (9) Exhaust gas absorption device.

Fig. 2 is the liquid-phase bubbling reaction. Liquid-phase bubbling reaction equipment: (1) Chlorine rotameter; (2) Buffering bottle; (3) Chlorination reactor (glass, homemade, gas distribution with a sand core); (4) Oil bathing beaker; (5) Temperature magnetic stirring heater; (6) Thermometer; (7) Glass spherical condenser; (8) Gas-liquid separator; (9) Sampling valve; (10) Low-temperature circulating pump; (11) Exhaust gas absorption device.

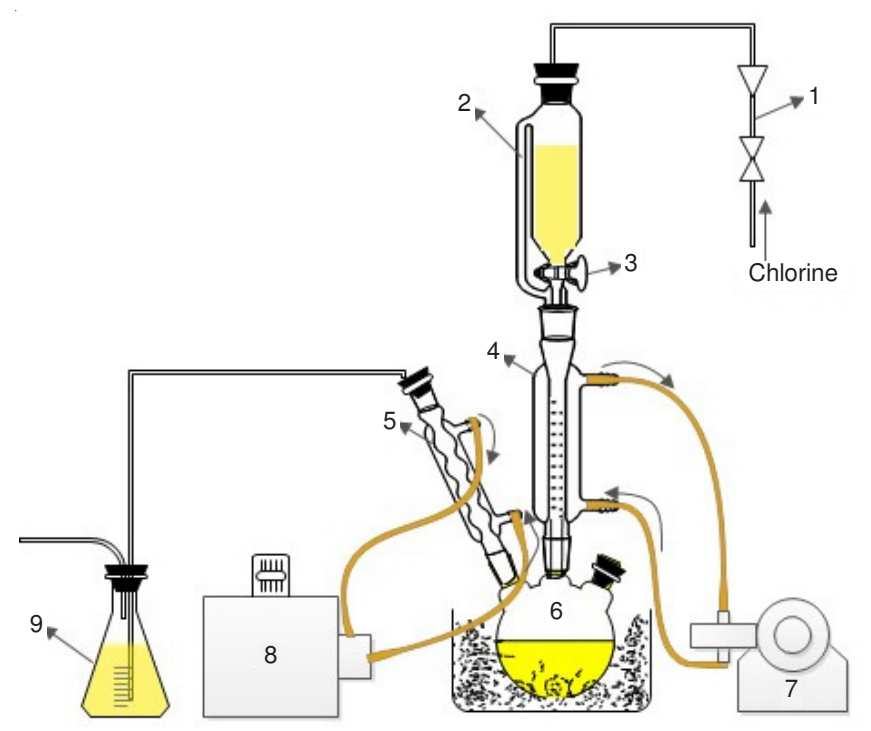

Fig. 1. Gas-liquid reaction equipment

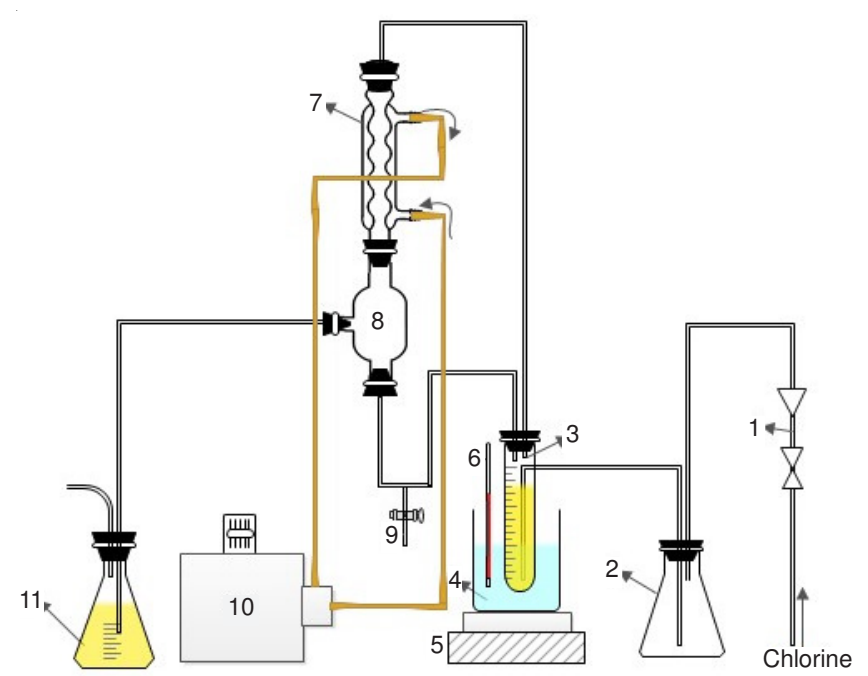

Fig. 2. Liquid-phase bubbling reaction equipment

\section{General procedure}

Gas-liquid mixed reaction of surface: A certain amount of acetic acid was filled into the balanced feeder and the hotwater circulating pump (water as medium) was opened after the water temperature reached the set temperature. After quantitative catalyst acetic anhydride was put into the balanced feeder, drip valve was opened and the speed of the drip was controlled precisely. After that, the chlorine should be passed into and the timer was started at the same time. After the reaction material was cooled in the reaction liquid receiving flask, the sample should be extracted and analyzed. The exhaust such as hydrogen chloride should be absorbed and purified by the cooling condenser device. Chlorine was stopped passing into after the reaction was finished.

The experimental temperature for the gas-liquid mixed reaction of surface was 60,80 and $90{ }^{\circ} \mathrm{C}$. Acetyl chloride 
concentration was from 25 to $98 \%$ (mass percentage), sample weighted $30 \mathrm{~g}$ and dropping time was from $1 \mathrm{~h}$ to $1.5 \mathrm{~h}$. The average residence time of packing material was from 15 to $25 \mathrm{~s}$. The excessive amount of chlorine, whose airflow was $22 \mathrm{~mL} / \mathrm{min}$, should flow into the reactor along with the material. Hot water was passed into the external cavity of the reactor, which was not only used for heating but also for heat extraction. The conversion ratio of the sample varied from 40 to $60 \%$.

Liquid phase bubbling reaction: A certain amount of acetic acid was filled into the chlorination reactor and the circulating pump (glycol solution as low temperature medium) was opened after the solution was cooled down to a set temperature. The quantitative catalyst was put into the chlorination reactor after it was heated to the specified temperature using a magnetic stirring heater. Then the chlorine was passed into and the timer was started at the same time. Rising steam was cooled and condensed in the condenser. Part of the condensate was taken out as samples, while the rest returned to the reactor. The overflow hydrogen chloride exhaust would be discharged into the air after it was absorbed by exhaust absorber, cooled by secondary condenser and absorbed by hydrochloric acid absorption bottle and alkaline washing bottle respectively. Chlorine was stopped passing into after the reaction was finished.

The temperature of liquid-phase bubbling reaction was $90{ }^{\circ} \mathrm{C}$ and the mass percentage of the added acetyl chloride was $25 \%$. Rising steam was cooled and condensed by the glass spherical condenser. The condensate was separated from the gas-liquid separator. Hydrogen chloride exhaust was absorbed and processed. Part of the condensate was taken out as samples, while the rest returned to the reactor.

Detection method: In this experiment, chloride solution was analyzed after esterification ${ }^{13,14}$ by GC2900 gas chromatograph. The chromatography working conditions are as follows: Column: $\varphi \mathrm{mm} \times 3000 \mathrm{~mm}$ stainless steel column; Detector: Thermal conductivity (TCD); Column temperature: $130{ }^{\circ} \mathrm{C}$; Vapourizer temperature: $180{ }^{\circ} \mathrm{C}$; TCD temperature: $150{ }^{\circ} \mathrm{C}$; Carrier gas $\left(\mathrm{H}_{2}\right)$ flow: $35-40 \mathrm{~mL} / \mathrm{min}$; Bridge flow : 80-100 $\mathrm{mA}$.

Qualitative analysis of each main component by means of the qualitative of standard compound; while quantitative analysis of each component is calculated by the absolute retention time and the correction area normalization method.

\section{RESULTS AND DISCUSSION}

Gas-liquid mixed reaction of surface: According to Fig. 3, no matter what ratio (acetyl chloride/acetic acid) of the reaction materials and no matter how much catalyst acetyl chloride was put. The dichloroacetic acid and chloroacetic acid yield molar ratio $\left(\mathrm{Y}^{=} / \mathrm{Y}^{-}\right)$decreased with the reaction temperature increasing. When the temperature increased, both the reaction rate and the conversion rate increased, which showed the concentration of chloroacetyl chloride in the system increased, while dichloroacetic acid and chloroacetic acid yield ratio decreased obviously. Therefore, the activation energy of acetyl chloride in the chlorination process is higher than that of chloroacetyl chloride and reaction rate of acetyl chloride with chlorine increases relatively with the increasing temperature.
At the same temperature, the value of $\mathrm{Y}^{=} / \mathrm{Y}^{-}$decreased with the acetic acid content increasing in the reactant. However, the trend slowed when the content of acetic acid was up to $40 \%$.

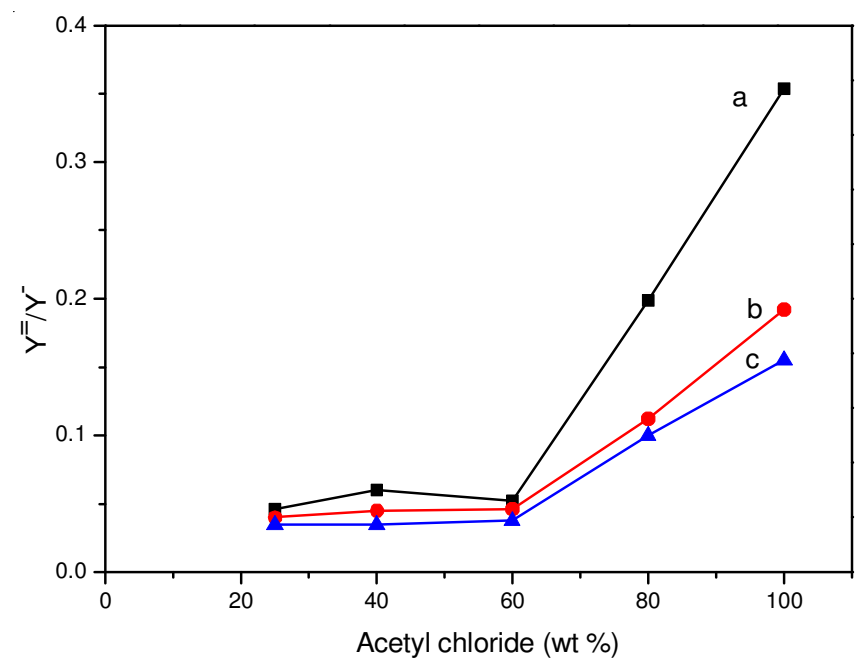

Fig. 3. Gas-liquid reaction for monochloroacetic acid and dichloroacetic acid; (a) $60{ }^{\circ} \mathrm{C}$; (b) $80{ }^{\circ} \mathrm{C}$; (c) $90^{\circ} \mathrm{C}$

At the same temperature, the yield ratio of dichloroacetic acid to chloroacetic acid reduced with the acetic acid content increasing in the reaction system, which showed a fast reaction rate of acetic acid with chloroacetyl chloride and the reaction was a fast reaction. The presence of acetic acid not only reduced the concentration of acetyl chloride in the reaction system relatively but also reduced the series reactions. This result was consistent with the previous conclusion.

When the conversion rate of acetic acid was elevated, the yield ratio of dichloroacetate increased faster, which was one of the late term of acetic acid chlorination reaction phenomenon. When the conversion rate of the acetic acid was higher, the concentration of acetic acid in the reaction system would be lower, then the mass transferring would be harder. If chlorine airflow of reaction medium and late term was consistent, chlorine would be in excess, which caused more chloroacetyl chloride further to convert into dichloroacetyl chloride and the generation rate of dichloroacetic acid would be faster and faster. Therefore, it is believed that the generation of dichloroacetic acid was in line with the series reactions mechanism. Parallel reactions are trimolecular reaction. According to a statistical point of view, there is a very slight chance for trimolecular to react.

Therefore, chlorine airflow should be controlled effectively throughout the process of the chlorination reaction so that the best reaction effect could be obtained in the case of the same amount of catalysts.

In late term of the reaction, the amount of acetyl chloride solution also decreased with the decreasing of the acetic acid concentration. At the same time, the generation rate of acetyl chloride slowed, while the dichloroacetic acid increased rapidly. Sometimes, chloroacetic acid concentration no longer increases but tends to reduce with the reduction of acetic acid concentration in the final stage of the late term of the reaction. However, at the same time, the concentration of the dichloroacetic acid has been increasing. 
Perhaps, there will be free radical in the late term of the reaction as the escaping of the catalysts and the increasing of the concentration of chlorine.

In order to solve this critical issue, the presence of the gas-phase reactor has been studied by means of liquid-phase bubbling reaction.

Liquid-phase bubbling reaction: According to Fig. 4, the generation rate of dichloroacetic acid increased quickly. Besides the amount of chlorine which has been discussed, a high concentration of acetyl chloride and chlorine can also cause reactions. In order to confirm this, in the liquid phase bubbling reaction, gas condensate was collected and the liquid and gas phase composition of the liquid phase reaction was analyzed. Therefore, it is known that the ratio of dichloroacetic acid to chloroacetic acid in the gas phase is many times greater than that in the liquid phase, which also shows that the generation amount of the dichloroacetyl chloride decreases due to the presence of acetic acid.

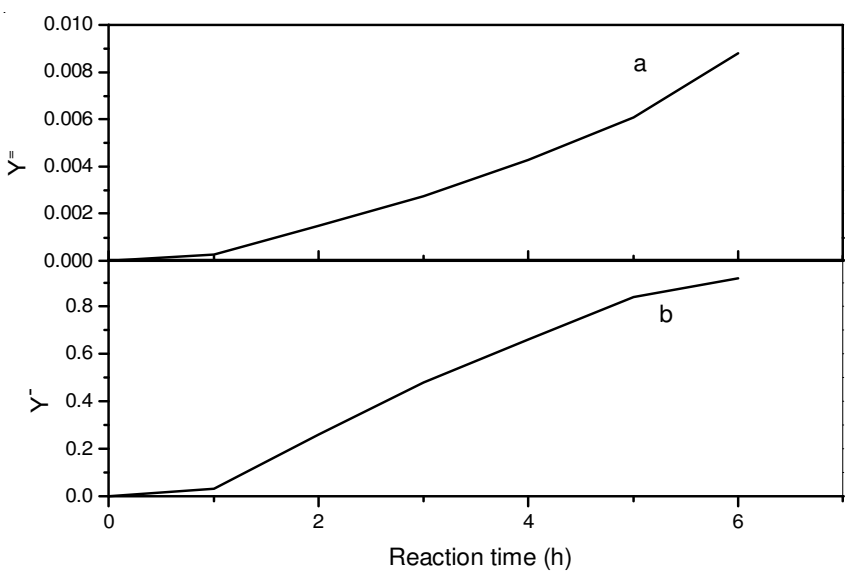

Fig. 4. Monochloroacetic acid yield and dichloroacetic acid yield of liquid reaction; (a) Dichloroacetic acid; (b) Chloroacetic acid

The amount of chlorine escaping from the liquid phase to the gas phase has been very little in the liquid-phase reaction process. Therefore, the results of the two reactions have great differences under the same concentration of acyl chloride and the byproduct dichloroacetic acid yield of liquid-phase bubbling reactor is much lower than that in the gas-liquid surface, which also shows that the acyl chloride in the gas phase is easy to react with chlorine and form series reactions.

Gas phase composition analysis of acetic acid chlorination reaction: According to the previous results, the late term reaction rate of the acetic acid chlorination reaction would slow down whether using sulfur or using acetic anhydride as catalyst, while the generation rate of the dichloroacetic acid would accelerate in the late term of the reaction. Many researchers have not been able to give a reasonable explanation for this phenomenon. It has been put forward that the generation of dichloroacetic acid was related with the gasphase reaction. In order to illustrate this point more effectively, concentration of reactants was estimated by distillation balance calculation for ternary composition, which was used to illustrate the source of acceleration for the generation of dichloroacetic acid.

The boiling point of the acetyl chloride is $50.8^{\circ} \mathrm{C}$. In the course of the reaction, the relative volatility of acetyl chloride has been increasing in the system with the acetic acid continuously converting to high boiling chloroacetic acid. The catalyst acetyl chloride rose into the gas phase with the releasing of hydrogen chloride exhaust airflow, which would result in the increasing of mole difference of acetyl chloride in the gas liquid two phase. As a consequence, the acetyl chloride might escape out of the system with the airflow if the condensation was ineffective. Maki-Arvela et al. ${ }^{12}$ studied the solubility of acetyl chloride in mixture containing varying amounts of chloroacetic acid (Table-1).

According to the data in Table-1, the solubility of acetyl chloride in the mixture has been decreasing with the increasing of chloroacetic acid content. The corresponding activity value could be calculated by means of Raoult's law $p_{i}=\gamma_{i} p_{i}^{0} x_{i}$. Therefore, The solution formed by acetyl chloride, acetic acid and chloroacetic acid is non-ideal solution.

As acetic acid, chloroacetic acid, dichloroacetic acid, acetyl chloride and chloroacetyl chloride existed in the reaction system, there were multimers of phase equilibria between acetic acid and chloroacetic acid, chloroacetic acid and chloroacetic acid, acetic acid and dichloroacetic acid. It was unknown whether there were dimer or trimer formation between acetyl chloride and acetic acid, chloroacetic acid. In addition, it was difficult to determine the activity coefficient of acetic acid and chloroacetic acid in the solution. There are mainly acetic acid and chloroacetic acid in the solution, in order to simplify the calculation, we make the following assumptions:

(1) Ignore dichloroacetic acid and other intermediates.

(2) The $\mathrm{HCl}$ gas continuously releases from the liquid phase and other components escaping with $\mathrm{HCl}$ should keep reciprocal balance with the liquid phase.

(3) Assume that the gas phase is an ideal gas.

\begin{tabular}{|c|c|c|c|c|c|c|}
\hline \multicolumn{7}{|c|}{$\begin{array}{c}\text { TABLE-1 } \\
\text { DENSITY OF COMPONENTS ON CHLORINATION }\end{array}$} \\
\hline \multirow{2}{*}{$\mathrm{P}_{\mathrm{AC}}$ (bar) } & $\mathrm{X}_{\mathrm{AC}, \mathrm{I}} / \mathrm{mol}-\%$ & \multirow[b]{2}{*}{$\gamma_{\mathrm{Ac}}$} & $\mathrm{X}_{\mathrm{AC}, \mathrm{l}} / \mathrm{mol}-\%$ & \multirow[b]{2}{*}{$\gamma_{\mathrm{Ac}}$} & $\mathrm{X}_{\mathrm{AC}, \mathrm{l}} / \mathrm{mol}-\%$ & \multirow[b]{2}{*}{$\gamma_{\mathrm{Ac}}$} \\
\hline & 30 mol-\% MCA* & & $50 \mathrm{~mol}-\% \mathrm{MCA}^{*}$ & & 70 mol- $\% \mathrm{MCA}^{*}$ & \\
\hline 0.74 & 2.82 & 7.56 & & & & \\
\hline 0.70 & 5.28 & 3.79 & & & & \\
\hline 0.92 & & & 1.80 & 14.63 & & \\
\hline 0.87 & & & 3.27 & 7.61 & & \\
\hline 1.09 & & & 3.40 & 9.17 & & \\
\hline 1.16 & & & & & 0.98 & 33.88 \\
\hline 1.50 & & & & & 2.02 & 21.25 \\
\hline
\end{tabular}


TABLE-2

GAS PARTIAL PRESSURE OF ACETYL CHLORIDE (FOR $5 \mathrm{~mol} \%$ )

\begin{tabular}{|c|c|c|c|c|c|c|}
\hline \multicolumn{3}{|c|}{$\begin{array}{l}\text { Liquid phase } \\
\text { x/mol } \%\end{array}$} & \multicolumn{3}{|c|}{$\begin{array}{l}\text { Gas phase } \\
\mathrm{y} / \mathrm{mol} \%\end{array}$} & \multirow{3}{*}{$\begin{array}{c}\begin{array}{c}\text { Sum of gas partial } \\
\text { pressure }\end{array} \\
\Sigma \mathrm{p}_{\mathrm{i}} \mathrm{mm} \mathrm{Hg}\end{array}$} \\
\hline $\mathrm{AcCl}^{*}$ & $\mathrm{AC}$ & MCA & $\mathrm{AcCl}$ & $\mathrm{AC}$ & MCA & \\
\hline $\mathrm{x}_{1}$ & $\mathrm{x}_{2}$ & $\mathrm{x}_{3}$ & $\mathrm{y}_{1}$ & $\mathrm{y}_{2}$ & $\mathrm{y}_{3}$ & \\
\hline \multirow{5}{*}{0.05} & 0.90 & 0.05 & 0.3238 & 0.6740 & 0 & 404.58 \\
\hline & 0.50 & 0.45 & 0.8735 & 0.1204 & 0.0062 & 1259.93 \\
\hline & 0.20 & 0.75 & 0.9745 & 0.0210 & 0.0451 & 2890.66 \\
\hline & 0.10 & 0.85 & 0.9902 & 0.0065 & 0.0032 & 4630.93 \\
\hline & 0.05 & 0.90 & 0.9945 & 0.0027 & 0.0028 & 5164.84 \\
\hline
\end{tabular}

TABLE-3

GAS PARTIAL PRESSURE OF ACETYL CHLORIDE (FOR 2 mol \%)

\begin{tabular}{|c|c|c|c|c|c|c|}
\hline \multicolumn{3}{|c|}{ Liquid phase } & \multicolumn{3}{|c|}{ Gas phase } & \multirow{3}{*}{$\begin{array}{c}\begin{array}{c}\text { Sum of } \\
\text { partial pressure }\end{array} \\
\Sigma \mathrm{p}_{\mathrm{i}} \mathrm{mmHg}\end{array}$} \\
\hline $\mathrm{AcCl}^{*}$ & $\mathrm{AC}$ & MCA & $\mathrm{AcCl}$ & $\mathrm{AC}$ & MCA & \\
\hline $\mathrm{x}_{1}$ & $\mathrm{x}_{2}$ & $\mathrm{x}_{3}$ & $\mathrm{y}_{1}$ & $\mathrm{y}_{2}$ & $\mathrm{y}_{3}$ & \\
\hline \multirow{5}{*}{0.02} & 0.98 & 0.00 & 0.1500 & 0.8500 & 0.0000 & 349.34 \\
\hline & 0.50 & 0.48 & 0.7363 & 0.2524 & 0.0116 & 600.00 \\
\hline & 0.28 & 0.70 & 0.9207 & 0.0693 & 0.0100 & 1223.82 \\
\hline & 0.10 & 0.88 & 0.9757 & 0.0161 & 0.0081 & 1879.95 \\
\hline & 0.03 & 0.95 & 0.9888 & 0.0040 & 0.0072 & 2279.23 \\
\hline
\end{tabular}

*Acetyl chloride

(4) The activity coefficient of acetic acid and chloroacetic acid in the mixture is 1 .

There were following relationship formulae for the gas vapour pressure and solution composition at some reaction temperature:

$$
\begin{gathered}
\mathrm{p}=\gamma_{1} \mathrm{p}_{1}^{0} \mathrm{x}_{1}+\gamma_{2} \mathrm{p}_{2}^{0} \mathrm{x}_{2}+\gamma_{3} \mathrm{p}_{3}^{0} \mathrm{x}_{3} \\
\mathrm{y}_{\mathrm{i}}=\gamma_{\mathrm{i}} \mathrm{p}_{\mathrm{i}}^{0} * \mathrm{x}_{\mathrm{i}} /\left(\gamma_{1} \mathrm{p}_{1}^{0} \mathrm{x}_{1}+\gamma_{2} \mathrm{p}_{2}^{0} \mathrm{x}_{2}+\gamma_{3} \mathrm{p}_{3}^{0} \mathrm{x}_{3}\right)
\end{gathered}
$$

The results were calculated and shown in Tables 2 and 3 according to the formula (2).

It can be seen from the data in the table above, when the conversion rate of acetic acid is $80 \%$, the molar ratio of acetic acid and chloroacetic acid of rising gases is only $1 \%$, while acetyl chloride account for $99 \%$. The above calculation may not be correct and the assumption may not be reasonable. However, it can be seen that the content of acetyl chloride in the liquid phase is only $5 \%$ when the conversion rate of acetic acid reach $70 \%$. The partial pressure of acetyl chloride is more than 1 atm, which show that it is not possible for the system to contain acetyl chloride more than $5 \%$ at $90^{\circ} \mathrm{C}$ under the operating pressure. Meanwhile, it can also explain that pressurized reaction is advantageous indirectly.

According to the assumptions and calculations for the chlorination process, the solubility of acetyl chloride in the liquid phase is very small when the conversion rate of the acetic acid is higher. At this time, the proportion of acetyl chloride in the rising gases was very high. In order to maintain the concentration of acetyl chloride in the liquid phase, pressurized operation must be implemented.

According to the calculation, the reaction system reduces the solubility of the acetyl chloride in the late term of the reaction, which causes the reaction rate to slow. Therefore, the higher reaction temperature causes the reaction rate to decline in the chlorination reaction process.
If chlorine is in excess in the late term of the reaction, gas phase reaction will be intensified. And series reactions in the gas phase will exacerbate the side effects, which cause the proportion of dichloroacetic acid to increase. As a result, data from many researchers show that the generation rate of dichloroacetic acid accelerates in the late term of the reaction.

\section{Conclusions}

(1) In the chlorination reaction of acetic acid, the solution is distilled off. The concentration of acetyl chloride in the gas phase is high due to the low boiling point of the acetyl chloride. As a consequence, the acetyl chloride concentration can reach $99 \%$ in the late term of the reaction. Such a high concentration of acetyl chloride can cause it to escape quickly with the escaping of hydrogen chloride in the exhaust gases. Therefore, the reaction rate slows and the amount of byproduct dichloroacetic acid increases.

(2) The reaction activation energy of acetyl chloride with chlorine is higher than that of chloroacetyl chloride with chlorine in the chlorination reaction. Therefore, if possible, the formation amount of the byproduct dichloroacetic acid will reduce with the reaction temperature increasing as high as possible.

(3) There are gas phase reaction and liquid phase reaction in the acetic acid chlorination reaction. The generation amount of the byproduct dichloroacetic acid is much lower in the liquid phase reaction than that in the gas reaction, which is due to the high concentration of acetic acid and the appropriate reaction mass transferring in the liquid phase reaction. The gas phase reaction is prone to series reactions, which make the amount of dichloroacetate increase.

(4) In order to improve the yield of chloroacetic acid, reducing the gas-phase reaction and increasing the concentration of acetyl chloride in the liquid phase are very important factors. 


\section{ACKNOWLEDGEMENTS}

Two of the authors, Jianwei Xue and Jianpeng Zhang are grateful to Taiyuan University of Technology, Shanxi, China for their encouragement and financial support, Institute of Fine Chemicals of Taiyuan University of Technology, Shanxi, China for providing the necessary facilities.

\section{REFERENCES}

1. G. Sioli, P.M. Spaziante and L. Giuffre, Hydrocarb. Process., 58, 111 (1979).

2. L. Guiffre, E. Montoneri and E. Tempesti, La Chimica e l'Industria, 63, 85 (1981).

3. P. Martikainen, T. Salmi, E. Paatero, L. Hummelstedt, P. Klein, H Damén and T. Lindroos, J. Chem. Technol. Biotechnol., 40, 259 (1987).
4. S. Kumar and J.K. Gehlawat, J. Chem. Tech. Biotechnol., 29, 353 (1979).

5. Y. Ogata and S. Watanabe, J. Org. Chem., 44, 2768 (1979).

6. Y. Ogata, T. Sugimoto and M. Inaishi, Bull. Chem. Soc. Japan., 52, 255 (1979).

7. T. Salmi, P. Martikainen, E. Paatero, L. Hummelstedt, H. Damén and T. Lindroos, Chem. Eng. Sci., 43, 1143 (1988).

8. P. Maki-Arvela, E. Paatero and T. Salmi, J. Chem. Tech. Biotechnol., 61, 1 (1994).

9. T. Salmi, E. Paatero and K. Fagerstolt, Chem. Eng. Sci., 48, 735 (1993).

10. T. Salmi, E. Paatero and K. Fagerstolt, IChemE (Part A), 71, 531 (1993).

11. M.F. Cui, X. Qiao, L.N. Wang and L. Huang, J. Nanjing Inst. Technol., 24, 24 (2002) (in Chinese).

12. P. Maki-Arvela, T. Salmi and E. Paatero, Ind. Eng. Chem. Res., 33, 2073 (1994).

13. C. Hu, M. Hashimoto, T. Okuhara and M. Misono, J. Catal., 143, 437 (1993).

14. J.W. Xue and C.L. Liu, Asian J. Chem., 20, 4859 (2008). 